\title{
Oscillations and integrability of the vorticity in the 3D NS flows
}

\author{
$\begin{array}{lll}\text { Y. Do } & \text { A. Farhat } & \text { Z. Grujić } \\ & \text { L. } & \mathrm{Xu}^{1}\end{array}$ \\ ${ }^{1}$ University of Virginia, ${ }^{2}$ Florida State University
}

October 19, 2018

\begin{abstract}
In the studies of the Navier-Stokes (NS) regularity problem, it has become increasingly clear that a more realistic path to improved a priori bounds is to try to break away from the scaling of the energy-level estimates in the realm of the blow-up-type arguments (the solution in view is regular/smooth up to the possible blow-up time) rather than to try to improve the regularity of Leray's weak solutions. The present article is a contribution in this direction; more precisely, it is shown-in the context of an algebraic/polynomial-type blow-up profile of arbitrary degree-that a very weak condition on the vorticity direction field (membership in a local bmo space weighted with arbitrary many logarithms)-suffices to break the energy-level scaling in the bounds on the vorticity. At the same time, the obtained bounds transform a 3D NS criticality scenario depicted by the macro-scale long vortex filaments into a no-singularity scenario.
\end{abstract}

Keywords: $L \ln ^{(k)} L$ Estimates; Vortex Stretching Term; Weighted BMO spaces; Pointwise Multipliers in BMO.

\section{Introduction}

The 3D Navier-Stokes (NS) equations read

$$
u_{t}+(u \cdot \nabla) u=\nu \triangle u-\nabla p+f,
$$

supplemented with the incompressibility condition $\operatorname{div} u=0$. A vector field $u$ denotes the velocity of the fluid, a scalar field $p$ the pressure, a positive constant $\nu$ the viscosity, and a vector field $f$ the external force. In what follows, for simplicity, the viscosity is set to $1, f$ taken to be a potential force and the spatial domain to be the whole space $\mathbb{R}^{3}$.

Taking the curl of the $(u, p)$ formulation yields the equations for the vorticity of the fluid $\omega$ $(\omega=\operatorname{curl} u)$,

$$
\omega_{t}+(u \cdot \nabla) \omega=\triangle \omega+(\omega \cdot \nabla) u .
$$

(Since we are on $\mathbb{R}^{3}$, the velocity can be recovered from the vorticity by the Biot-Savart law, and one obtains a closed system for $\omega$.) In this setting, the only piece of data is the initial condition.

The left-hand side represents the transport of the vorticity by the velocity, the first term on the right-hand side is the diffusion, and the second term is the vortex-stretching term. The vortexstretching term $(\omega \cdot \nabla) u$ is responsible for amplification of the vorticity magnitude in 3D NS flows and possible formation of singularities (it is identically zero in $2 \mathrm{D}$ ). 
The NS regularity problem is super-critical, i.e., there is a scaling gap between any regularity class/criterion and the corresponding a priori bound (with respect to the unique scaling leaving the NS equations invariant). Moreover, the scaling gap has been of the fixed size; all the regularity classes are (at best) scaling-invariant, while all the a priori bounds have been on the scaling level of the Leray's energy class $L^{\infty}\left(0, T ; L^{2}\right)$. Somewhat unexpectedly, a very recent work (Bradshaw, Farhat, and Grujić [3]) introduced a mathematical framework based on the suitably defined scale of spatial intermittency/sparseness of the regions of intense vorticity-and motivated by the computational simulations of turbulent flows-in which the regularity class is still scaling invariant, but the a priori bound is algebraically/polynomially better than the energy-level bound (this is in the context of a blow-up-type argument; i.e., one studies an initially smooth solution in the vicinity of the first possible blow-up time).

There are two main classical approaches in the study of the NS regularity problem; one is to try to improve the regularity of the Leray's weak solutions (or some other class of weak solutions), and the other one is to study the initially smooth/regular flows approaching a possible singularity (a blow-up-type argument). Given that there is an increasing evidence that Leray's solutions may not be unique (see, for example, a very recent work of Buckmaster and Vicol [5]), and the fact that regular solutions are necessarily unique, the second avenue seems more realistic. In addition, the aforementioned reduction of the scaling gap ([3]) took place within the realm of the blow-up-type arguments.

The main goal of this article is to show that-in the context of a blow-up-type argument-one can escape the energy-level scaling of the vorticity a priori bounds under a very weak condition on the oscillations of the vorticity direction field $\xi$. We were motivated by the work of Bradshaw and Grujić [2] where it was shown that as long as $\xi$ is uniformly-in-time bounded in the logarithmically weighted space of the functions of bounded mean oscillations $\widetilde{b m o} \frac{1}{|\operatorname{logr}|}$, the vorticity magnitude stays bounded in the Zygmund space $L \log L$ breaking the energy-level scaling $L^{1}$. A notable feature of this result is that the space $\widehat{b m o} \frac{1}{\log r}$ allows for discontinuities (e.g., discontinuities of $\sin \log |\log r|$-type). At the same time, the question arose of whether it was possible to break the energy-level scaling in the vorticity bounds under a weaker assumption on $\xi$ (allowing for stronger discontinuities), or whether there is a natural obstruction and the space $\widetilde{b m o} \frac{1}{|\operatorname{logr}|}$ is optimal within this framework.

Unfortunately, there is an obstruction. Namely, one of the main ingredients in [2] is the CoifmanRochberg BMO-estimate on the logarithm of the maximal function ([7]) which has no analogue in the weighted $B M O$-spaces. In particular, in the case where the weight is a $k$-fold log-composite (the case of the most interest for applications to the 3D NSE), several types of counterexamples are presented in the Appendix. It turned out that the obstruction disappears if one restricts the considerations to the case where the function of interest (its magnitude) exhibits an algebraic/polynomialtype singularity (there is no restriction on the degree/strength of the singularity). Fortunately, in the realm of the 3D NSE, an algebraic/polynomial type of the blow-up profile (for the vorticity magnitude) is consistent with the current NS theory, and-in this context-it is indeed possible to generalize the result from [2], i.e., replace the original $\xi$-condition $\widetilde{b m o} \frac{1}{|\log r|}$ with the class of $\xi$-conditions

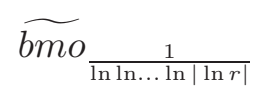


where the number of the log-composites can be an arbitrary positive integer, and still obtain the bound on the vorticity magnitude escaping the energy-level scaling.

In addition, the estimates on the vorticity magnitude obtained here yield improved estimates on the distribution function of the vorticity sufficient to transform a geometric criticality scenario presented in Dascaliuc and Grujić [9] into a regularity scenario.

The paper is organized as follows. Section 2 presents several results on weighted local $B M O \mathrm{~s}$ and maximal functions; in particular, a 'dynamic' generalization of the Coifman-Rochberg estimate in the setting of the algebraic/polynomial-type singularity is presented. Section 3 contains the main result.

\section{Results on Weighted BMOs and Maximal Functions}

In this section, we list and prove some results in harmonic analysis that will be applied in the next section. Henceforth, for a set $S$ and a function $f, f(S)$ will denote the average value of $f$ on $S$, $f(S)=f_{s} f=\frac{1}{|S|} \int_{S} f$.

Lemma 2.1. Let $\varphi$ be a suitable weight function and $\delta>0$ sufficiently small (depending on $\varphi$ ). For any $f \in L^{1}$ define

$$
\|f\|_{\widehat{b m o \varphi}}:=\|f\|_{L^{1}}+\sup _{|Q|<\delta} \frac{1}{\varphi(r)} f_{Q}|f(x)-f(Q)| d x .
$$

Suppose $\phi:(0, \infty) \rightarrow[0, \infty)$ is increasing near 0 , and let $\phi_{*}$ be defined by

$$
\phi_{*}(t)=\int_{\min (\delta, t)}^{\delta} \frac{\phi(s)}{s} d s .
$$

Then

$$
\|f g\|_{\widetilde{b m o}} \lesssim\|f\|_{\widetilde{b m o_{\phi}}}\left(\|g\|_{\infty}+\|g\|_{b m o_{\left(\phi_{*}\right)-1}}\right)
$$

(Here, $\widetilde{b m o}=\widetilde{b m o_{1}}$.)

Proof. We slightly modify the proof of Theorem 2 in Janson [10] as follows. Let $Q=Q\left(x_{0}, r\right)$ be any cube with center $x_{0}$ and side length $r<\frac{\delta}{2}$. Then, as shown in [10],

$$
|f(Q)| \lesssim\|f\|_{\widetilde{b m o_{\phi}}} \int_{r}^{\delta} \frac{\phi(t)}{t} d t \lesssim \phi_{*}(r)\|f\|_{\widetilde{b m o_{\phi}}} .
$$

The following string of inequalities is then easily verified.

$$
\begin{aligned}
& (f g)(Q) \lesssim f_{Q\left(x_{0}, r\right)}|g||f-f(Q)| d x+f_{Q\left(x_{0}, r\right)}|f||g-g(Q)| d x \\
& \lesssim\|g\|_{\infty} f_{Q\left(x_{0}, r\right)}|f-f(Q)| d x+|f(Q)| f_{Q\left(x_{0}, r\right)}|g-g(Q)| d x \\
& \lesssim \phi(r)\|f\|_{\widetilde{b m o_{\phi}}}\|g\|_{\infty}+\|f\|_{\widetilde{b m o_{\phi}}} \phi_{*}(r) f_{Q\left(x_{0}, r\right)}|g-g(Q)| d x \\
& \lesssim\|f\|_{\widetilde{b m o_{\phi}}}\|g\|_{\infty}+\|f\|_{\widetilde{b m o_{\phi}}}\|g\|_{b m o_{\left(\phi_{*}\right)-1}} \text {. }
\end{aligned}
$$


Lemma 2.2. For an $0<r<1$ and a function $f$ define the following incarnation of the maximal operator: $M_{r}(f)=\left(M\left(|f|^{r}\right)\right)^{1 / r}$ where the usual maximal operator is defined as

$$
M(f)(x)=\sup _{x \in Q} \frac{1}{|Q|} \int_{Q}|f(y)| d y
$$

where the supremum is taken over all cubes containing $x$. Let $\left\{f_{t}\right\}$ be a family of functions satisfying

$$
\begin{aligned}
& \text { 1. }\left\{f_{t}\right\} \subset C^{1}\left(\mathbb{R}^{n} \backslash\{0\}\right) \\
& \text { 2. } \sup _{t}\left|\nabla f_{t}\right| \lesssim|x|^{-\ell} \\
& \text { 3. } \inf _{t}\left|f_{t}\right| \gtrsim 1 .
\end{aligned}
$$

Then there exists $r \in(0,1)$ such that $\nabla M_{r}\left(f_{t}\right)$ exists almost everywhere and

$$
\sup _{t}\left|\nabla M_{r}\left(f_{t}\right)\right| \lesssim|x|^{-k}
$$

where $k$ depends only on $r$ and $\ell$.

Proof. Let $f$ be a member $\left\{f_{t}\right\}$. Note that $\nabla M_{r}(f)$ exists almost everywhere because the maximal function maps Hölder continuous function to Hölder continuous function and in particular Lipschitz function to Lipschitz function (cf. Buckley [4]). Let $g=|f|^{r}$. We choose $r$ small enough so that $|g| \lesssim|x|^{-m}$ and $m<n-1$. Fix an $x \in Q$ and let $\tilde{Q}=(y-x)+Q$. Then $y \in \tilde{Q}$ if and only if $x \in Q$, and for any $Q$ containing $x$

$$
\begin{aligned}
f_{Q} g d z & \leq f_{Q} g d z-f_{\tilde{Q}} g d z+f_{\tilde{Q}} g d z \\
& \leq \sup _{y \in \tilde{Q}}\left|f_{Q} g d z-f_{\tilde{Q}} g d z\right|+\sup _{y \in \tilde{Q}} f_{\tilde{Q}} g d z \\
& =\sup _{x \in Q}\left|f_{Q} g d z-f_{\tilde{Q}} g d z\right|+\sup _{y \in Q} f_{Q} g d z .
\end{aligned}
$$

Consequently

$$
|M(g)(x)-M(g)(y)| \leq \sup _{x \in Q}\left|f_{Q} g d z-f_{\tilde{Q}} g d z\right|
$$

Now fix an $x \neq 0$ and suppose $y$ is close enough to $x$. Let $Q_{0}$ be the cube centered at 0 with side length $|x| / 4$. If $Q \cap\left(2 Q_{0}\right)=\emptyset$, then for $y$ close enough to $x$

$$
\left|f_{Q} g d z-f_{\tilde{Q}} g d z\right| /|x-y| \leq f_{Q} \frac{|g(z+y-x)-g(z)|}{|x-y|} d z \leq f_{Q}\left|\nabla g\left(\xi_{z, x, y}\right)\right| d z \lesssim|x|^{-\ell} .
$$

If $Q \cap\left(2 Q_{0}\right) \neq \emptyset$, then $\operatorname{diam}(Q) \geq|x| / 2$ (the diameter of $Q$ ) and

$$
\left|f_{Q} g d z-f_{\tilde{Q}} g d z\right| /|x-y| \leq(|Q||x-y|)^{-1}\left(\int_{(Q \Delta \tilde{Q}) \cap Q_{0}} g d z+\int_{(Q \Delta \tilde{Q}) \cap Q_{0}^{c}} g d z\right) .
$$


For the first part of the right hand side, we have estimates

$$
(|Q||x-y|)^{-1} \int_{(Q \Delta \tilde{Q}) \cap Q_{0}^{c}} g d z \lesssim|x|^{-1} f_{(Q \Delta \tilde{Q}) \cap Q_{0}^{c}} g d z \lesssim|x|^{-1}|x|^{-(\ell-1) r} \lesssim|x|^{-(\ell-1) r-1} .
$$

For the second part we have

$$
(|Q||x-y|)^{-1} \int_{(Q \Delta \tilde{Q}) \cap Q_{0}} g d z \lesssim|x|^{-1} f_{(Q \Delta \tilde{Q}) \cap\left(2 Q_{0}\right)} g d z \lesssim|x|^{-1} f_{(Q \Delta \tilde{Q}) \cap\left(2 Q_{0}\right)}|z|^{-m} d z .
$$

Note that the maximal average of $|z|^{-m}$ takes place when $Q \Delta \tilde{Q}$ contains the origin, in which case $(Q \Delta \tilde{Q}) \cap\left(2 Q_{0}\right)$ is a flat cube inside $2 Q_{0}$ whose height is at most $|x-y|$. The largest possible average is attained when $y \rightarrow x$, i.e. the flat cube shrinks to an area in $\mathbb{R}^{n-1}$. So

$$
\begin{aligned}
(|Q||x-y|)^{-1} \int_{(Q \Delta \tilde{Q}) \cap Q_{0}} g d z & \lesssim|x|^{-1} f_{\left(2 Q_{0}\right) \cap \mathbb{R}^{n-1}}|\tilde{z}|^{-m} d \tilde{z} \\
& \lesssim|x|^{-1}|x|^{1-n}|x|^{-m+n-1} \lesssim|x|^{-m-1} .
\end{aligned}
$$

To sum up, for some fixed $k$ and for $y$ close enough to $x$, we have

$$
\left|f_{Q} g d z-f_{\tilde{Q}} g d z\right| /|x-y| \lesssim|x|^{-k} \text {. }
$$

Combining the two cases yields

$$
|\nabla M(g)(x)| \lesssim \sup _{\text {all directions } y} \lim _{y \rightarrow x}|M(g)(x)-M(g)(y)| /|x-y| \lesssim|x|^{-k_{1}} .
$$

Note that the second assumption implies $M(g) \lesssim|x|^{-\ell^{\prime}}$ for some $\ell^{\prime}$. Consequently,

$$
\left|\nabla M_{r}(f)(x)\right| \lesssim|x|^{-k_{2}} \quad \text { for some } k_{2}
$$

finishing the argument.

Lemma 2.3. Suppose a family of differentiable functions $\left\{f_{t}\right\}_{0<t<T}$ is compactly supported in $B(0,1)$ (open ball with radius 1 centered at 0 ) and

1. $\left|f_{t}(x)\right| \gtrsim \frac{1}{|x|^{\epsilon_{1}}+(T-t)^{\epsilon_{2}}}$ in $B\left(0, \frac{1}{2}\right)$ for some $\epsilon_{1}, \epsilon_{2}>0$

2. $\sup _{t}\left|\nabla f_{t}(x)\right| \lesssim \frac{1}{|x|^{\ell_{1}}}$ for some $\ell_{1}>0$

3. $\sup _{x}\left|\nabla f_{t}(x)\right| \lesssim \frac{1}{(T-t)^{\ell_{2}}}$ for some $\ell_{2}>0$.

Set $\phi_{*}(x)=\ln ^{(k)}(|\ln x|)=\log \log \ldots \log |\log x|($ for some $k \in \mathbb{N})$ and $\phi(x)=-x \phi_{*}^{\prime}(x)$ for small $x$. Then, for a suitable $r \in(0,1)$, $\sup _{t}\left\|\phi_{*}\left(M_{r} f_{t}\right)\right\|_{\widetilde{b m o_{\phi}}} \lesssim 1$. 
Proof. Lemma 2.2 and the second assumption imply

$$
\sup _{t}\left|\nabla M_{r} f_{t}\right| \lesssim \frac{1}{|x|^{\ell_{1}}}
$$

for some $r \in(0,1)$. In fact $\ell_{1}$ can be different from the one given in the assumption, but for convenience we still denote it by $\ell_{1}$. Following a similar argument as in Lemma 2.2, we deduce that

$$
\left|M\left(\left|f_{t}\right|^{r}\right)(x)-M\left(\left|f_{t}\right|^{r}\right)(y)\right| \leq\left.\sup _{x \in Q}\left|f_{Q}\right| f_{t}(z)\right|^{r} d z-f_{\tilde{Q}}\left|f_{t}(z)\right|^{r} d z \mid
$$

and that

$$
\begin{aligned}
\frac{\left|M\left(\left|f_{t}\right|^{r}\right)(x)-M\left(\left|f_{t}\right|^{r}\right)(y)\right|}{|x-y|} & \leq\left.\sup _{x \in Q}\left|f_{Q}\right| f_{t}(z)\right|^{r} d z-f_{\tilde{Q}}\left|f_{t}(z)\right|^{r} d z|/| x-y \mid \\
& \lesssim \sup _{x \in Q} f_{Q} \frac{\left.|| f_{t}\right|^{r}(z+y-x)-\left|f_{t}\right|^{r}(z) \mid}{|x-y|} d z \\
& \left.\lesssim \sup _{x \in Q} f_{Q}|\nabla| f_{t}\right|^{r}\left(\xi_{z, x, y}\right) \mid d z
\end{aligned}
$$

Therefore $\left.\left|\nabla M\left(\left|f_{t}\right|^{r}\right)(x)\right| \lesssim \sup _{x \in Q} f_{Q}|\nabla| f_{t}\right|^{r}\left(\xi_{z, x, y}\right) \mid d z$. Having in mind the first assumption implies $\inf _{t, x}\left|f_{t}\right|(x) \gtrsim 1$, we see

$$
\left|\nabla M\left(\left|f_{t}\right|^{r}\right)(x)\right| \lesssim \sup _{x \in Q} f_{Q}|\nabla| f_{t}\left|\left(\xi_{z, x, y}\right)\right| d z
$$

The second assumption implies that $\sup _{t}\left|f_{t}\right| \lesssim|x|^{-\ell_{1}+1}$, so $\sup _{x \in \partial B\left(0, \frac{1}{2}\right)}\left|f_{t}\right| \lesssim 1$. Then the third assumption implies that

$$
\sup _{x \in B\left(0, \frac{1}{2}\right)} M\left(f_{t}\right) \lesssim \frac{1}{(T-t)^{\ell_{2}}} .
$$

Combining (2.6) and (2.5) with the third assumption we obtain

$$
\sup _{x}\left|\nabla M_{r} f_{t}\right| \lesssim \frac{1}{(T-t)^{\ell_{2}^{\prime}}} \quad \text { for some } \ell_{2}^{\prime} .
$$

Now we divide the proof into three cases:

If $\operatorname{dist}(0, Q) \gtrsim(\operatorname{diam}(Q))^{\gamma}$ with $\gamma<1 / \ell_{1}$, note that by $(2.4)$

$$
\begin{aligned}
f_{Q} \phi_{*}\left(M_{r} f_{t}\right) d x-\inf _{Q} \phi_{*}\left(M_{r} f_{t}\right) & \lesssim \sup _{Q} \phi_{*}\left(M_{r} f_{t}\right)-\inf _{Q} \phi_{*}\left(M_{r} f_{t}\right) \\
& \lesssim \sup _{Q}\left|\phi_{*}^{\prime}\left(M_{r} f_{t}\right) \cdot \nabla M_{r} f_{t}\right| \cdot \operatorname{diam}(Q) \\
& \lesssim \phi_{*}^{\prime}\left(\inf _{Q} M_{r} f_{t}\right) \cdot \sup _{Q}\left|\nabla M_{r} f_{t}\right| \cdot \operatorname{diam}(Q)
\end{aligned}
$$


From the first assumption we know $\inf _{Q} M_{r} f_{t} \gtrsim 1$, so $\phi_{*}^{\prime}\left(\underset{Q}{\inf } M_{r} f_{t}\right) \lesssim 1$, and

$$
\begin{aligned}
f_{Q} \phi_{*}\left(M_{r} f_{t}\right) d x-\inf _{Q} \phi_{*}\left(M_{r} f_{t}\right) & \lesssim(\operatorname{dist}(0, Q))^{-\ell_{1}} \cdot \operatorname{diam}(Q) \\
& \lesssim|Q|^{\left(1-\gamma \ell_{1}\right) / n} \lesssim \phi(|Q|) .
\end{aligned}
$$

If $T-t \gtrsim(\operatorname{diam}(Q))^{\delta}$ with $\delta<1 / \ell_{2}$, similarly, by $(2.7)$

$$
\begin{aligned}
f_{Q} \phi_{*}\left(M_{r} f_{t}\right) d x-\inf _{Q} \phi_{*}\left(M_{r} f_{t}\right) & \lesssim \phi_{*}^{\prime}\left(\inf _{Q} M_{r} f_{t}\right) \cdot \sup _{Q}\left|\nabla M_{r} f_{t}\right| \cdot \operatorname{diam}(Q) \\
& \lesssim(T-t)^{-\ell_{2}} \operatorname{diam}(Q) \\
& \lesssim|Q|^{\left(1-\delta \ell_{2}\right) / n} \lesssim \phi(|Q|) .
\end{aligned}
$$

The last inequality follows from the fact that $\left(1-\delta \ell_{2}\right) / n>0$ and $|x|^{\left(1-\delta \ell_{2}\right) / n} \ll \phi(|x|)$ when $|x|$ is sufficiently small.

If $\operatorname{dist}(0, Q) \lesssim(\operatorname{diam}(Q))^{\gamma}$ and $T-t \lesssim(\operatorname{diam}(Q))^{\delta}$, we divide the proof into two subcases. Case (i): if $0 \notin Q$, then by the first assumption we have

$$
\begin{aligned}
\inf _{Q} M_{r} f_{t} & \geq \inf _{Q}\left|f_{t}\right| \gtrsim \frac{1}{(\operatorname{dist}(0, Q)+\operatorname{diam}(Q))^{\epsilon_{1}}+(T-t)^{\epsilon_{2}}} \\
& \gtrsim(\operatorname{diam}(Q))^{-\min \left\{\gamma \epsilon_{1}, \delta \epsilon_{2}\right\}} \gtrsim|Q|^{-\epsilon_{3}} .
\end{aligned}
$$

This implies (by Jensen's inequality and the mean value theorem)

$$
\begin{aligned}
f_{Q} \phi_{*}\left(M_{r} f_{t}\right) d x-\inf _{Q} \phi_{*}\left(M_{r} f_{t}\right) & \lesssim\left(\phi_{*} \circ \exp \right)\left(f_{Q} \ln \left(M_{r} f_{t}\right) d x\right)-\left(\phi_{*} \circ \exp \right)\left(\inf _{Q} \ln \left(M_{r} f_{t}\right)\right) \\
& \lesssim\left(\phi_{*} \circ \exp \right)^{\prime}\left(\xi_{Q}\right)\left(f_{Q} \ln \left(M_{r} f_{t}\right) d x-\inf _{Q} \ln \left(M_{r} f_{t}\right)\right)
\end{aligned}
$$

where $\inf _{Q} \ln \left(M_{r} f_{t}\right) \leq \xi_{Q} \leq f_{Q} \ln \left(M_{r} f_{t}\right) d x$. Note that $\left(\phi_{*} \circ \exp \right)^{\prime}$ is decreasing near 0 , and that by Coifman and Rochberg [7] we have $f_{Q} \ln \left(M_{r} f_{t}\right) d x-\inf _{Q} \ln \left(M_{r} f_{t}\right) \lesssim 1$; consequently

$$
f_{Q} \phi_{*}\left(M_{r} f_{t}\right) d x-\inf _{Q} \phi_{*}\left(M_{r} f_{t}\right) \lesssim\left(\phi_{*} \circ \exp \right)^{\prime}\left(\ln |Q|^{-\epsilon_{3}}\right) \lesssim \phi(|Q|)
$$

Case (ii): if $0 \in Q$, then

$$
\inf _{Q} M_{r} f_{t} \geq \inf _{Q}\left|f_{t}\right| \gtrsim \frac{1}{(\operatorname{diam}(Q))^{\epsilon_{1}}+(T-t)^{\epsilon_{2}}} \gtrsim(\operatorname{diam}(Q))^{-\min \left\{\epsilon_{1}, \delta \epsilon_{2}\right\}}
$$

which leads back to the proof of Case (i). 


\section{Main Result}

This section is devoted to our main result which is formulated in the following theorem.

Theorem 3.1. Let $u$ be a Leray solution to the $3 D N S E$ on $\mathbb{R}^{3} \times(0, \infty)$. Assume that the initial vorticity $\omega_{0} \in L^{1} \cap L^{2}$, and that $T>0$ is the first (possible) blow-up time. Suppose that

1. for some choice of the parameters $\epsilon_{1}, \epsilon_{2}, \ell_{1}, \ell_{2}>0$ and some neighborhood $U$ of 0 ,

- $|\omega(x, t)| \gtrsim \frac{1}{|x|^{\epsilon_{1}}+(T-t)^{\epsilon_{2}}}$

- $\sup _{t<T}|\nabla \omega(x, t)| \lesssim \frac{1}{|x|^{\ell_{1}}}$

- $\sup _{x \in U}|\nabla \omega(x, t)| \lesssim \frac{1}{(T-t)^{\ell_{2}}}$

(in particular, $\epsilon_{1}, \epsilon_{2}$ can be arbitrarily small and $\ell_{1}, \ell_{2}$ can be arbitrarily large),

2. $\operatorname{setting} \varphi(r)=\left(\ln { }^{(k)}|\ln r|\right)^{-1}=(\ln \ln \ldots \ln |\ln r|)^{-1}$ and $\xi(t, x)=\omega(t, x) /|\omega(t, x)|$,

$$
\sup _{t \in(0, T)}\|(\psi \xi)(t, \cdot)\|_{\widetilde{b m o} \varphi}<\infty
$$

Then

$$
\sup _{t \in(0, T)} \int \psi(x) w(x, t) \Phi(w(x, t)) d x<\infty .
$$

where $w=\sqrt{\exp ^{(k+1)}(1)+|\omega|^{2}}$ and $\Phi(r)=\ln ^{(k)}(|\ln r|)$.

Remark 3.2. Condition 1. is a description of the algebraic/polynomial nature of the blow-up profile (this is consistent with the current 3D NS theory) suitable for our purposes; note that the degree is arbitrary, i.e., there is no restriction on the strength of the singularity. Condition 2 . is a very mild (the number of log-composites is arbitrary) condition on the oscillation of the vorticity direction allowing for a wide range of discontinuities.

Proof. Recall that (cf. Bradshaw and Grujić [2])

$$
\partial_{t} w-\Delta w+(u \cdot \nabla) w \leq \omega \cdot \nabla u \cdot \frac{\omega}{w} .
$$

Let $f(w)=\Phi(w)+w \Phi^{\prime}(w)$. Then

$$
\nabla f(w)=f^{\prime}(w) \nabla w=\left(\Phi^{\prime}(w)+\left(w \Phi^{\prime}(w)\right)^{\prime}\right) \nabla w,
$$

and one can show that

$$
0 \leq w\left(\Phi^{\prime}(w)+\left(w \Phi^{\prime}(w)\right)^{\prime}\right) \leq 1
$$

for all $w$; consequently, $w f^{\prime}(w)$ is non-negative and bounded. Multiplying the initial inequality by $\psi f$ yields

$$
\psi f(w) \partial_{t} w-\psi f(w) \Delta w+\psi f(w)(u \cdot \nabla) w \leq \psi f(w) \omega \cdot \nabla u \cdot \frac{\omega}{w}
$$


Time-derivative:

$$
\begin{aligned}
\partial_{t}(\psi w \Phi(w)) & =\psi \partial_{t}(w \Phi(w)) \\
& =\psi\left(\Phi(w) w_{t}+w \Phi^{\prime}(w) w_{t}\right) \\
& =\psi f(w) w_{t}
\end{aligned}
$$

Laplacian:

$$
\begin{aligned}
-\Delta w \psi f(w) & =-\nabla \cdot(\psi f(w) \nabla w)+\nabla(\psi f(w)) \cdot \nabla w \\
& =-\nabla \cdot(\psi f(w) \nabla w)+f(w) \nabla \psi \cdot \nabla w+\psi \nabla f(w) \cdot \nabla w \\
& =-\nabla \cdot(\psi f(w) \nabla w)+f(w) \nabla \psi \cdot \nabla w+\psi f^{\prime}(w)|\nabla w|^{2} .
\end{aligned}
$$

Note that after taking the integral $-\nabla \cdot(\psi f(w) \nabla w)$ vanishes, and since $\psi f^{\prime}(w)|\nabla w|^{2}$ is always non-negative, it can be dropped.

Advection:

$$
(u \cdot \nabla) w \psi f(w)=\psi u \cdot \nabla F(w)=\nabla \cdot(\psi u F(w))-(u \cdot \nabla \psi) F(w)
$$

where $F$ is an antiderivative of $f$ and $|F(w)| \lesssim|w|^{1+\epsilon}$ for arbitrary small $\epsilon$. The first term vanishes after taking the integral.

Vortex-stretching:

$$
\begin{aligned}
\omega \cdot \nabla u \cdot \frac{\omega}{w} \psi f(w) & =\omega \cdot \nabla u \cdot \xi \psi f(w)+\omega \cdot \nabla u \cdot\left(\frac{\omega}{w}-\frac{\omega}{|\omega|}\right) \psi f(w) \\
& =\omega \cdot \nabla u \cdot \xi \psi \Phi(w)+\omega \cdot \nabla u \cdot \xi \psi w \Phi^{\prime}(w)+\omega \cdot \nabla u \cdot\left(\frac{\omega}{w}-\frac{\omega}{|\omega|}\right) \psi f(w) .
\end{aligned}
$$

In the spirit of [2], we arrive at

$$
I(\tau) \equiv \int \psi(x) w(x, \tau) \Phi(w(x, \tau)) d x \leq I(0)+c \int_{0}^{\tau} \int_{x} \omega \cdot \nabla u \cdot \psi \xi \Phi(w) d x d t+J(\tau)
$$

where

$$
\begin{aligned}
J= & -\int_{0}^{\tau} \int_{x} f(w) \nabla \psi \cdot \nabla w d x d t+\int_{0}^{\tau} \int_{x}(u \cdot \nabla \psi) F(w) d x d t \\
& +\int_{0}^{\tau} \int_{x} \omega \cdot \nabla u \cdot \xi \psi w \Phi^{\prime}(w) d x d t+\int_{0}^{\tau} \int_{x} \omega \cdot \nabla u \cdot\left(\frac{\omega}{w}-\frac{\omega}{|\omega|}\right) \psi f(w) d x d t \\
= & : J_{1}+J_{2}+J_{3}+J_{4} .
\end{aligned}
$$

Note that $|f(w)| \lesssim w^{\epsilon}$ for arbitrary small $\epsilon$; Hölder inequality with $1 / p+1 / q=1$ and Lions [11, Theorem 3.7] with $L^{q}\left(\mathbb{R}^{N} \times(0, T)\right)(q<4 / 3)$ then yields

$$
J_{1} \lesssim\left(\int_{t} \int_{x}\left|w f^{\prime}(w)+f(w)\right|^{p}\right)^{1 / p}\left(\int_{t} \int_{x}|\nabla w|^{q}\right)^{1 / q} \lesssim\left(\int_{t} \int_{x} w\right)^{1 / p}<\infty .
$$


Turning to $J_{2}$, Hölder and Sobolev inequalities imply

$$
\begin{aligned}
J_{2} & \lesssim \int_{0}^{\tau}\|F(w) \nabla \psi\|_{2}\|u\|_{2} \lesssim \sup _{t}\|u\|_{2} \int_{0}^{\tau}\|F(w) \nabla \psi\|_{2} \\
& \lesssim \int_{0}^{\tau}\|F(w) \nabla \psi\|_{2} \lesssim \int_{0}^{\tau}\|\nabla(F(w) \nabla \psi)\|_{\frac{6}{5}} \\
& \lesssim \int_{0}^{\tau}\|F(w)\|_{\frac{6}{5}}+\|f(w) \nabla w\|_{\frac{6}{5}} \\
& \lesssim \int_{0}^{\tau}\|F(w)\|_{\frac{6}{5}}+\int_{0}^{\tau}\|f(w)\|_{p^{\prime}}\|\nabla w\|_{q^{\prime}}
\end{aligned}
$$

where $1 / p^{\prime}+1 / q^{\prime}=5 / 6$ and $6 / 5<q^{\prime}<4 / 3$. Note that

$$
\|F(w)\|_{\frac{6}{5}} \lesssim\|w\|_{2}^{\frac{5}{3}} \text { and }\|f(w)\|_{p^{\prime}} \lesssim\|w\|_{1}^{1 / p^{\prime}} ;
$$

hence, by using the result in Constantin [8] (also see Lions [11]),

$$
J_{2} \lesssim \int_{0}^{\tau}\|w\|_{2}^{2}+\left(\sup _{t}\|w\|_{1}\right)^{1 / p^{\prime}} \int_{0}^{\tau}\|\nabla w\|_{q^{\prime}} \lesssim \int_{t} \int_{x}|\nabla u|^{2}+\int_{t} \int_{x}|\nabla w|^{q^{\prime}}<\infty .
$$

Regarding $J_{3}$ and $J_{4}$, it suffices to observe that

$$
\left|\xi w \Phi^{\prime}(w)\right| \lesssim 1 \text { and }\left|\frac{\omega}{w}-\frac{\omega}{|\omega|}\right| \lesssim|\omega|^{-2}
$$

respectively.

This completes the a priori boundedness of $J$, and we can turn our attention to the remaining (leading order) vortex-stretching term.

On one hand, by the convexity of $\ln ^{(k)} x$ (for large $x$ ) we deduce

$$
\left|\Phi\left(\mathcal{M}_{r} w\right)-\Phi(w)\right| \leq\left|\log \left(\mathcal{M}_{r} w\right)-\log w\right|=\log \left(\mathcal{M}_{r} w / w\right) \leq \mathcal{M}_{r} w / w-1
$$

this yields

$$
\int_{0}^{\tau} \int_{x} \omega \cdot \nabla u \cdot \psi \xi\left(\Phi(w)-\Phi\left(\mathcal{M}_{r} w\right)\right) d x d t \leq \int_{0}^{\tau} \int_{x}|\nabla u|\left(\mathcal{M}_{r} w-\omega\right) d x d t
$$

which is a priori bounded by Hölder inequality and the fact that $\mathcal{M}_{r}$ is bounded on $L^{2}$. On the other hand, $\mathcal{H}^{1}-B M O$ duality, Div-Curl Lemma (cf. Coifman et al. [6]), Lemma 2.1 and Condition 2. (3.1) combine to

$$
\begin{aligned}
\int_{0}^{\tau} \int_{x} \omega \cdot \nabla u \cdot \psi \xi \Phi\left(\mathcal{M}_{r} w\right) d x d t & \lesssim \int_{0}^{\tau}\|\omega \cdot \nabla u\|_{\mathcal{H}^{1}}\left\|\psi \xi \Phi\left(\mathcal{M}_{r} w\right)\right\|_{B M O} d t \\
& \lesssim \int_{0}^{\tau}\|\omega \cdot \nabla u\|_{\mathcal{H}^{1}}\left\|\psi \xi \Phi\left(\mathcal{M}_{r} w\right)\right\|_{\widetilde{b m o}} d t \\
& \lesssim \int_{0}^{\tau}\|\omega\|_{2}\|\nabla u\|_{2}\left(\|\psi \xi\|_{\infty}+\|\psi \xi\|_{\widetilde{b m o} o_{\varphi}}\right)\left\|\Phi\left(\mathcal{M}_{r} w\right)\right\|_{\widetilde{b m o} \phi} d t \\
& \lesssim \sup _{t \in(0, T)}\left\|\Phi\left(\mathcal{M}_{r} w\right)\right\|_{\widetilde{b m o_{\phi}}} \int_{0}^{\tau} \int_{x}|\nabla u|^{2} d x d t
\end{aligned}
$$


where $\phi$ satisfies $\varphi=\left(\phi_{*}\right)^{-1}$ (here, we are referring to the notation of Lemma 2.3). Consequently, Lemma 2.3 paired with Condition 1. imply

$$
\sup _{t \in(0, T)}\left\|\Phi\left(\mathcal{M}_{r} w\right)\right\|_{\widetilde{b m o} \phi}<\infty
$$

This completes the proof.

Remark 3.3. Instead of imposing a restriction on time dynamics of the vorticity near the possible singular time $T$, one can assume that the singular profile at $t=T$ has a somewhat regular shape, e.g. an almost radial symmetry plus an almost monotonicity plus an almost convexity, or satisfying the assumptions of Lemma A.1 below, and assume that the mean oscillations leading to the blow-up time are not worse than the mean oscillations of the singular profile.

\section{A Appendix}

Lemma A.1. Suppose a Lebesgue measurable function $f$ is compactly supported in a neighborhood of 0 and

1. $|f| \gtrsim|x|^{-\epsilon}$ for some $\epsilon$

2. $|\nabla f| \lesssim|x|^{-\ell}$ for some $\ell$.

Then, $\phi_{*}\left(M_{r} f\right) \in \widetilde{\text { bmo }_{\phi}}$ where $\phi$ and $\phi_{*}$ are given in Lemma 2.3.

Proof. The proof is similar to Lemma 2.3, utilizing Lemma 2.2.

The rest of the Appendix is devoted to demonstrating that Condition 1. in Theorem 3.1 is essentially optimal within the mathematical framework utilized.

Lemma A.2. Let $f: \mathbb{R} \rightarrow \mathbb{C}$ be differentiable such that

1. $f$ is even on $[-1,1]$

2. there exists a constant constant $C>0$ such that for every $y \in[-1,1]$ and $|x| \geq|y|$

$$
\left|f^{\prime}(x)\right| \leq C\left|f^{\prime}(y)\right|
$$

3. $\left|x f^{\prime}(x)\right| \lesssim \phi(x)$ for all $x \in[0,1]$.

Then for every interval I of length at most 1 we have

$$
\frac{1}{|I|} \int_{I}\left|f-f_{I}\right| \lesssim \phi(|I|)
$$

In particular, if we assume that $f \in L^{1}$, then $f \in \widetilde{b^{\prime} o_{\phi}}$. 
Proof. Let $I=\left[a_{I}, b_{I}\right]$. Consider the case when $I \subset[0, \infty)$ first. Then by the triangle inequality

$$
\begin{aligned}
\frac{1}{|I|} \int_{I}\left|f(x)-f_{I}\right| d x & \leq \frac{1}{|I|^{2}} \int_{I^{2}}|f(x)-f(y)| d x d y \\
& =\frac{2}{|I|^{2}} \iint_{a_{I} \leq x \leq y \leq b_{I}}|f(x)-f(y)| d x d y \\
& \leq \frac{2}{|I|^{2}} \iint_{a_{I} \leq x \leq y \leq b_{I}} \int_{x}^{y}\left|f^{\prime}(t)\right| d t d x d y \\
& =\frac{2}{|I|^{2}} \int_{I}\left|f^{\prime}(t)\right|\left(t-a_{I}\right)\left(b_{I}-t\right) d t \\
& \lesssim \frac{1}{|I|} \int_{I}\left|f^{\prime}\left(t-a_{I}\right)\right|\left(t-a_{I}\right) d t
\end{aligned}
$$

(using (A.1) we have $\left|f^{\prime}(t)\right| \lesssim\left|f^{\prime}\left(t-a_{I}\right)\right|$, while clearly $0 \leq b_{I}-t \leq|I|$ )

$$
=\frac{1}{|I|} \int_{0}^{|I|}\left|f^{\prime}(t)\right| t d t \lesssim \frac{1}{|I|} \int_{0}^{|I|} \phi(t)|I| d t \leq \phi(|I|)
$$

using the monotonicity of $\phi$.

The case when $I \subset[-\infty, 0]$ is entirely similar.

Now consider the remaining case when $a_{I}<0<b_{I}$. Without loss of generality we may assume that $\left|a_{I}\right| \leq\left|b_{I}\right|$, and it suffices to show that for some constant $c$ we have

$$
\frac{1}{|I|} \int_{I}|f-c| \lesssim \phi(|I|)
$$

(This would imply $(1 /|I|) \int_{I}\left|f-f_{I}\right|=(1 /|I|) \int_{I}\left|(f-c)-(f-c)_{I}\right| \leq(2 /|I|) \int_{I}|f-c| \lesssim \phi(|I|)$.)

Set $c=\frac{1}{b_{I}} \int_{0}^{b_{I}} f$. Since $|I| \leq 1, I \subset[-1,1]$, and $f$ being even on $[-1,1]$ implies

$$
\frac{1}{|I|} \int_{I}|f-c| \lesssim \frac{1}{|I|} \int_{0}^{b_{I}}|f-c| \lesssim \frac{1}{b_{I}} \int_{0}^{b_{I}}|f-c|
$$

which reduces this case to the case $I \subset[0, \infty)$ considered above.

Lemma A.3. Let $f:(0,1) \rightarrow(0, \infty)$ be differentiable, decreasing and integrable near 0 , and such that for every open interval $I \subset(0,1)$ of sufficiently small length we have

$$
\frac{1}{|I|} \int_{I}\left|f-f_{I}\right| \lesssim \phi(|I|)
$$

1. Assume that for $x$ sufficiently near $0\left|x f^{\prime}(x)\right| \gtrsim \psi(x)$ where $\psi$ is essentially increasing nonnegative on $(0,1]$. Then $\psi(x) \lesssim \phi(4 x)$ for $x$ sufficiently near 0 .

2. Assume that in addition $f$ is also near 0 . Then $\left|x f^{\prime}(x)\right| \lesssim \phi(x)$ near 0 . 
Proof. (1) Let $I=(0, T)$ where $T<1$ is sufficiently small. By the mean value theorem $f(\alpha)=$ $T^{-1} \int_{0}^{T} f(x) d x$ for some $\alpha \in(0, T)$, and since $f^{\prime}(t)<0$ we have

$$
\begin{aligned}
T \phi(T) & \gtrsim \int_{I}\left|f(x)-f_{I}\right| d x=\int_{0}^{\alpha}|f(x)-f(\alpha)| d x+\int_{\alpha}^{T}|f(x)-f(\alpha)| d x \\
& =\int_{0}^{\alpha} \int_{x}^{\alpha}\left|f^{\prime}(t)\right| d t d x+\int_{\alpha}^{T} \int_{\alpha}^{x}\left|f^{\prime}(t)\right| d t d x \\
& =\int_{0}^{\alpha}\left|f^{\prime}(t)\right| \int_{0}^{t} d x d t+\int_{\alpha}^{T}\left|f^{\prime}(t)\right| \int_{t}^{T} d x d t \\
& =\int_{0}^{\alpha}\left|f^{\prime}(t)\right| t d t+\int_{\alpha}^{T}\left|f^{\prime}(t)\right|(T-t) d x d t=J_{1}+J_{2} .
\end{aligned}
$$

If $\alpha<T / 2$, the second term can be bounded from below as

$$
J_{2} \gtrsim \int_{T / 2}^{c T / 2}\left|f^{\prime}(t)\right| t d t \gtrsim \int_{T / 2}^{c T / 2} \psi(t) d t \gtrsim_{c} T \psi(T / 2)
$$

using the essentially increasing property of $\psi$, and if $\alpha>T / 2$, the first term as

$$
J_{1} \gtrsim \int_{T / 4}^{c T / 4} \psi(t) d t \gtrsim T \psi(T / 4) .
$$

Consequently,

$$
T \phi(T) \gtrsim \int_{I}\left|f(x)-f_{I}\right| d x \gtrsim T \psi(T / 4)
$$

which implies the desired estimate.

(2) We proceed similarly as above and obtain (considering the two cases for $\alpha$ )

$$
\begin{aligned}
T \phi(T) & \gtrsim \min \left\{\int_{0}^{T / 2}\left|f^{\prime}(t)\right| t d t, \int_{T / 2}^{T}\left|f^{\prime}(t)\right|(T-t) d t\right\} \\
& \gtrsim T \min \left\{\int_{T / 4}^{T / 2}\left|f^{\prime}(t)\right| d t, \int_{T / 2}^{3 T / 4}\left|f^{\prime}(t)\right| d t\right\} .
\end{aligned}
$$

Utilizing that $f^{\prime}<0$ and $f^{\prime}$ increasing near 0 (because of convexity), we have that $\left|f^{\prime}\right|$ is decreasing near 0 ; hence, for $T$ sufficiently small,

$$
T \phi(T) \gtrsim T^{2} \min \left\{\left|f^{\prime}(T / 2)\right|,\left|f^{\prime}(3 T / 4)\right|\right\} \gtrsim T^{2}\left|f^{\prime}(T)\right| .
$$

Thus $x\left|f^{\prime}(x)\right| \lesssim \phi(x)$ for $x$ near 0 completing the proof.

Theorem A.4. There exists a sequence $\left\{f_{n}\right\} \subset L^{p} \cap C_{c}^{\infty}([-1,1])$ such that

$$
\left\|\phi_{*}\left(M\left(f_{n}\right)\right)\right\|_{\widetilde{b m o_{\phi}}} \rightarrow \infty
$$

where $\phi_{*}(x)=\ln ^{(k)}(|\ln x|), k \geq 1$ and $\phi(x)=-x \phi_{*}^{\prime}(x)$ for small $x$. 
Remark A.5. It is worth noting that the above result fails when $k=0$, i.e., when $\phi_{*}(x)=|\ln x|$ for small $x$ and-consequently- $\phi=1$ (this follows directly from Coifman and Rochberg [7]). As a matter of fact, this is the main reason that the case $k \geq 1$ is more intricate than the case $k=0$ considered in Bradshaw and Grujić [2].

Proof. Let us discuss the problem in one dimension (examples for higher dimensions can be obtained by rotating the one-dimensional examples). Start with the function

$$
f(x)=s^{-\delta} \cdot \chi_{[-s, s]}+\ell_{1} \cdot \chi_{[-s-\epsilon,-s)}+\ell_{2} \cdot \chi_{(s, s+\epsilon]}+C
$$

where $s, \delta, \epsilon>0, C>1$ and $\ell_{1}, \ell_{2}$ are two straight lines connecting the constant pieces in the continuous way, and mollify the sharp corners at $\pm s, \pm(s+\epsilon)$ (we call the modified function $f$ ) in a way ensuring that $f \equiv s^{-\delta}+C$ on $[-s, s]$ and $f \equiv C$ outside $[-\epsilon-s, s+\epsilon]$. Then for $x>s+\epsilon$ $($ or $x<-s-\epsilon)$

$$
M(f)(x)=\frac{A+s^{-\delta}(1-\eta(x) / 2) \eta(x) \epsilon}{x+\eta(x) \epsilon}+C
$$

where $A=\int_{-\epsilon-s}^{s+\epsilon} f d x$ and $\eta(x)=\frac{2(x-s)}{x+\sqrt{x^{2}+2 \epsilon(x-s)}}$. Let

$$
h=\phi_{*}(M(f)) .
$$

In what follows, we will show that by sending the parameters $s$ and $\delta$ to 0 in a suitable fashion, the weighted BMO norm of $h$ tends to infinity. More precisely, we are after the sequences $s_{n}$ and $\delta_{n}$ and some intervals $I_{n}=\left(k s_{n}, r s_{n}\right)($ where $1 \lesssim k, r \lesssim 1)$ such that

$$
\left|I_{n}\right| \inf _{x \in I_{n}}\left|h_{n}^{\prime}(x)\right| / \phi\left(\left|I_{n}\right|^{-1}\right) \rightarrow \infty
$$

Then Lemma A.3-(ii) would imply that $\left\|h_{n}\right\|_{\widetilde{b m o_{\phi}}} \rightarrow \infty$. In order to apply Lemma A.3 we will show that $h_{n}$ is monotone and convex on $I_{n}$ and also analyze the asymptotic behavior of the quantity $\left|I_{n}\right| \inf _{x \in I_{n}}\left|h_{n}^{\prime}(x)\right| / \phi\left(\left|I_{n}\right|^{-1}\right)$.

A straightforward calculation gives

$$
\begin{aligned}
\frac{d}{d x} M(f) & =\frac{s^{-\delta} \epsilon\left(\gamma^{\prime}-\gamma \gamma^{\prime}\right)(x+\gamma \epsilon)-\left(1+\gamma^{\prime} \epsilon\right)\left(A+s^{-\delta}(1-\gamma / 2) \gamma \epsilon\right)}{(x+\gamma \epsilon)^{2}} \\
\frac{d^{2}}{d x^{2}} M(f) & =\epsilon F\left(\gamma, \gamma^{\prime}, \gamma^{\prime \prime}, x, \epsilon, A, s\right)+A(x+\gamma \epsilon)^{-4}
\end{aligned}
$$

where $\gamma^{\prime}(x)=2-\frac{2(x-s)\left(1+(x+s) / \sqrt{x^{2}+2 \epsilon(x-s)}\right)}{x+\sqrt{x^{2}+2 \epsilon(x-s)}}$. When $x$ is comparable to $s$ (i.e. $\left.x \approx k s\right), \gamma^{\prime}$ and $\gamma^{\prime \prime}$ are bounded. In addition, for a fixed $s, F$ is bounded when $\epsilon \rightarrow 0$. Hence, if $x$ is comparable to $s$ and $\epsilon$ is small enough $(\epsilon \ll s)$, then

$$
M(f) \approx A x^{-1}, \quad \frac{d}{d x} M(f) \approx-A x^{-2}, \quad \frac{d^{2}}{d x^{2}} M(f) \approx A x^{-4}
$$


consequently for sufficiently small $s$ and $x$ ( $x$ is comparable to $s$ )

$$
\begin{aligned}
h^{\prime} & =\phi_{*}^{\prime}\left(M\left(f_{n}\right)\right) \frac{d}{d x} M\left(f_{n}\right) \approx-\left(\ln A x^{-1}\right)^{-1} x^{-1}<0, \\
h^{\prime \prime} & =\phi_{*}^{\prime}\left(M\left(f_{n}\right)\right) \frac{d^{2}}{d x^{2}} M\left(f_{n}\right)+\phi_{*}^{\prime \prime}\left(M\left(f_{n}\right)\right)\left(\frac{d}{d x} M\left(f_{n}\right)\right)^{2} \\
& \approx\left(\ln A x^{-1}\right)^{-1} x^{-3}-\left(\ln A x^{-1}\right)^{-2} x^{-2}>0 .
\end{aligned}
$$

Now choose $k, r(1 \lesssim k, r \lesssim 1)$ such that on $I=(k s, r s)$ the above requirements are satisfied (and Lemma A.3 applies). For a fixed $\delta$, sending $s \rightarrow 0$

$$
|I| \inf _{x \in I}\left|h^{\prime}(x)\right| / \phi\left(|I|^{-1}\right) \approx s\left(\ln \left(s^{1-\delta} s^{-1}\right)\right)^{-1} s^{-1} /\left(\ln s^{-1}\right)^{-1} \approx \delta^{-1} .
$$

Summarizing, for each $n$ we can choose $I_{n}$ such that $\left|I_{n}\right| \inf _{x \in I_{n}}\left|h_{n}^{\prime}(x)\right| / \phi\left(\left|I_{n}\right|^{-1}\right.$ ) is close to $\delta_{n}^{-1}$ (with $\left.\delta_{n} \rightarrow 0\right)$ and thus

$$
\sup _{n}\left|I_{n}\right| \inf _{x \in I_{n}}\left|h_{n}^{\prime}(x)\right| / \phi\left(\left|I_{n}\right|^{-1}\right)=\infty
$$

Corollary A.6. There exists a sequence $\left\{f_{n}\right\} \subset C_{c}^{\infty}([-1,1])$ such that $f_{n} \rightarrow f$ pointwise (also in $\left.L^{p}\right), \phi_{*}\left(M_{\delta}(f)\right) \in L^{p} \cap \widehat{b m o}_{\phi}$ and $|f| \gtrsim|x|^{-\ell}$ but

$$
\left\|\phi_{*}\left(M_{\delta}\left(f_{n}\right)\right)\right\|_{\widetilde{b m o_{\phi}}} \rightarrow \infty
$$

where $\phi_{*}(x)=\ln ^{(k)}(|\ln x|)(k \geq 1)$ and $\phi(x)=-x \phi_{*}^{\prime}(x)$ for small $x$.

Proof. As in the previous theorem, it suffices to consider the one-dimensional setting. Consider the mollified version of the function

$$
f(x)=s^{-\delta} \cdot \chi_{[-s, s]}+t^{-\ell} \cdot \chi_{[-t, t]}+|x|^{-\ell} \cdot \chi_{\mathbb{R} \backslash[-t, t]}, \quad t \gg s
$$

(and call it $f$ ) such that for $s+\epsilon<x<t$

$$
M(f)=\frac{A+s^{-\delta}(1-\gamma(x) / 2) \gamma(x) \epsilon}{x+\gamma(x) \epsilon}+t^{-\ell}
$$

where $\gamma$ is as in Theorem A.4 and $A \approx s^{1-\delta}$. The rest of the argument is very similar to that of Theorem A.4, except that when sending $s$ and $\delta$ to 0 we let $s$ decrease much faster compared to $\delta$ because we want $A x^{-1}$ to dominate $t^{-\ell}$. A suitable choice is given by $s=e^{-1 / t}$ and $\delta=\sqrt{t}$. Then, if $x$ is comparable to $s$ (i.e. $x \approx k s$ ) we have

$$
A x^{-1} / t^{-\ell} \approx t^{\ell} s^{-\delta} \approx t^{\ell} e^{1 / \sqrt{t}} \rightarrow \infty
$$

and the same idea as in Theorem A.4 applies. 
Corollary A.7. For any $\ell>n$, there exists a (infinite) collection $\left\{f_{t}\right\} \subset C_{c}^{\infty}([-1,1])$ such that

$$
\left\|f_{t}\right\|_{L^{1}} \lesssim 1, \quad\left|\nabla f_{t}\right| \lesssim|x|^{-\ell}
$$

but $\left\|\phi_{*}\left(M\left(f_{t}\right)\right)\right\|_{\widetilde{b m o}_{\phi}} \rightarrow \infty$.

Proof. Consider a suitably mollified version of

$$
f(x)=s^{-\delta} \cdot \chi_{[-s, s]}+\left(s^{-\delta}-s^{1-\ell}+|x|^{1-\ell}\right) \chi_{(s, b]}+\left(s^{-\delta}-s^{1-\ell}+|x|^{1-\ell}\right) \chi_{[-b,-s)}+C
$$

where $b$ is determined by $s^{-\delta}-s^{-\ell}+b^{-\ell}=0$. Note that if $\ell>1$ and $\delta$ is sufficiently small then $b-s \ll s$ and $f \equiv C$ outside $[-b, b]$. Similarly as in Theorem A.4, for $x>s+b$ (or $x<-s-b$ )

$$
M(f) \approx A x^{-1}, \quad \frac{d}{d x} M(f) \approx-A x^{-2}, \quad \frac{d^{2}}{d x^{2}} M(f) \approx A x^{-4}
$$

where $A=\int_{-b-s}^{s+b} f d x$. Setting $h=\phi_{*}(M(f))$, one can show $h$ is monotone and convex on some interval $I=(k s, r s)(1 \lesssim k, r \lesssim 1)$ and

$$
|I| \inf _{x \in I}\left|h^{\prime}(x)\right| / \phi\left(|I|^{-1}\right) \approx s\left(\ln \left(s^{1-\delta} s^{-1}\right)\right)^{-1} s^{-1} /\left(\ln s^{-1}\right)^{-1} \approx \delta^{-1} .
$$

Finally, we choose $f_{s(t), \delta(t)}$ with $s(t), \delta(t) \rightarrow 0$ and $I_{s(t)}$ such that

$$
\left|I_{s}\right| \inf _{x \in I_{s}}\left|h_{s(t), \delta(t)}^{\prime}(x)\right| / \phi\left(\left|I_{s}\right|^{-1}\right) \approx \delta^{-1} \rightarrow \infty
$$

Lemma A.3 then yields $\left\|\phi_{*}\left(M\left(f_{s(t), \delta(t)}\right)\right)\right\|_{\widetilde{b m o}_{\phi}} \rightarrow \infty$. In addition, note that-by construction- $\left\{f_{s, \delta}\right\}$ is bounded in $L^{1}$ and its gradients bounded by $|x|^{-\ell}$.

Theorem A.8. For any $\alpha<n$ and $0<\alpha<\beta$, there exists $f \in L^{p} \cap C^{1}\left(\mathbb{R}^{n} \backslash\{0\}\right)$ such that $|x|^{-\alpha} \lesssim|f| \lesssim|x|^{-\beta}$ but

$$
\phi_{*}\left(M_{\delta}(f)\right) \notin \widetilde{b m o}_{\phi} .
$$

Proof. For simplicity set $n=1$ and $\delta=1$. Let $f$ be a suitable mollification $($ on $(0, \infty))$ of

$$
x^{-\alpha} \cdot \chi_{\mathbb{R} \backslash \cup I_{i}}+\sum_{i} a_{i}^{-\beta} \cdot \chi_{I_{i}}
$$

where $I_{i}=\left[a_{i}, b_{i}\right]$ are disjoint (e.g. $a_{n}=2^{-2^{n}}$ ) and $\left|I_{i}\right| \approx a_{i}^{\gamma_{i}}$ with $\gamma_{i} \rightarrow \infty$ and $\left|I_{i}\right| \ll\left(a_{i}-b_{i+1}\right)$. Then for $b_{i+1} \ll x<a_{i}$

$$
(M f)(x) \approx \frac{\left|I_{i}\right| a_{i}^{-\beta}+\int_{x}^{a_{i}} t^{-\alpha} d t}{b_{i}-x}
$$


(one can show that the average of $f$ on $\left[x, a_{i}\right]$ is increasing when $x \rightarrow a_{i}$ by differentiation). A direct computation when $a_{i}-x \approx\left|I_{i}\right|$ then gives

$$
\begin{aligned}
\frac{d}{d x}(M f)(x) & =\frac{-x^{-\alpha}\left(b_{i}-x\right)+\left(\left|I_{i}\right| a_{i}^{-\beta}+(1-\alpha)\left(a_{i}^{1-\alpha}-x^{1-\alpha}\right)\right)}{\left(b_{i}-x\right)^{2}} \\
& \approx \frac{-a_{i}^{-\alpha}\left(a_{i}-x+\left|I_{i}\right|\right)+\left(\left|I_{i}\right| a_{i}^{-\beta}+a_{i}^{-\alpha}\left(a_{i}-x\right)\right)}{\left|I_{i}\right|^{2}}=\frac{a_{i}^{-\beta}-a_{i}^{-\alpha}}{\left|I_{i}\right|}
\end{aligned}
$$

Let $g=\phi_{*}(M(f))$; then (for $\left.a_{i}-x \approx\left|I_{i}\right|\right)$

$$
\begin{aligned}
\left|g^{\prime}\right| & =|\phi(M(f))||M(f)|^{-1}\left|\frac{d}{d x}(M f)(x)\right| \\
& \gtrsim\left|\phi\left(a_{i}^{-\beta}\right)\right| \cdot a_{i}^{\beta} \cdot a_{i}^{-\beta}\left|I_{i}\right|^{-1} \approx\left|\phi\left(a_{i}^{-\beta}\right)\right|\left|I_{i}\right|^{-1} .
\end{aligned}
$$

Consequently, for some small interval $J_{i}\left(\left|J_{i}\right| \approx\left|I_{i}\right|\right)$ which is close to $a_{i}$ we have

$$
\begin{aligned}
\left|J_{i}\right| \inf _{x \in J_{i}}\left|g^{\prime}(x)\right| / \phi\left(\left|J_{i}\right|^{-1}\right) & \approx\left|J_{i}\right|\left|\phi\left(a_{i}^{-\beta}\right)\right|\left|I_{i}\right|^{-1} / \phi\left(\left|J_{i}\right|^{-1}\right) \\
& \approx\left|\phi\left(a_{i}^{-\beta}\right)\right| / \phi\left(\left|I_{i}\right|^{-1}\right) \approx\left|\phi\left(a_{i}^{-\beta}\right)\right| / \phi\left(a_{i}^{-\gamma_{i}}\right) \\
& \gtrsim\left|\ln a_{i}^{-\gamma_{i}}\right| /\left|\ln a_{i}^{-\beta}\right| \approx \gamma_{i} / \beta \rightarrow \infty .
\end{aligned}
$$

Lemma A.3 then implies $g=\phi_{*}(M(f)) \notin \widetilde{\operatorname{bmo}_{\phi}}$.

\section{References}

[1] Z. Bradshaw and Z. Grujić. Blow-up scenarios for the 3D Navier-Stokes equations exhibiting sub-criticality with respect to the scaling of one-dimensional local sparseness. J. Math. Fluid Mech., 16(2):321-334, 2014. ISSN 1422-6928. URL https://doi.org/10.1007/s00021-013-0155-0.

[2] Z. Bradshaw and Z. Grujić. A spatially localized $L \log L$ estimate on the vorticity in the 3D NSE. Indiana Univ. Math. J., 64(2):433-440, 2015. ISSN 0022-2518. URL https://doi.org/10.1512/iumj.2015.64.5496.

[3] Z. Bradshaw, A. Farhat, and Z. Grujić. An algebraic reduction of the 'scaling gap' in the Navier-Stokes regularity problem. URL https://arxiv.org/abs/1704.05546

[4] Stephen M. Buckley. Is the maximal function of a Lipschitz function continuous? Ann. Acad. Sci. Fenn. Math., 24(2):519-528, 1999. ISSN 1239-629X.

[5] T. Buckmaster and V. Vicol. Nonuniqueness of weak solutions to the Navier-Stokes equation. URL https://arxiv.org/abs/1709.10033 
[6] R. Coifman, P.-L. Lions, Y. Meyer, and S. Semmes. Compensated compactness and Hardy spaces. J. Math. Pures Appl. (9), 72(3):247-286, 1993. ISSN 0021-7824.

[7] R. R. Coifman and R. Rochberg. Another characterization of BMO. Proc. Amer. Math. Soc., 79(2):249-254, 1980. ISSN 0002-9939. URL https://doi.org/10.2307/2043245.

[8] Peter Constantin. Navier-Stokes equations and area of interfaces. Comm. Math. Phys., 129(2): 241-266, 1990. ISSN 0010-3616. URL http://projecteuclid.org/euclid.cmp/1104180744.

[9] R. Dascaliuc and Z. Grujić. Vortex stretching and criticality for the three-dimensional Navier-Stokes equations. J. Math. Phys., 53(11):115613, 9, 2012. ISSN 0022-2488. URL https://doi.org/10.1063/1.4752170.

[10] S. Janson. On functions with conditions on the mean oscillation. Ark. Mat., 14(2):189-196, 1976. ISSN 0004-2080. URL https://doi.org/10.1007/BF02385834.

[11] Pierre-Louis Lions. Mathematical topics in fluid mechanics. Vol. 1, volume 3 of Oxford Lecture Series in Mathematics and its Applications. The Clarendon Press, Oxford University Press, New York, 1996. ISBN 0-19-851487-5. Incompressible models, Oxford Science Publications. 